\title{
Data Processing for a High Resolution Preclinical PET Detector Based on Philips DPC Digital SiPMs
}

David Schug, Jakob Wehner, Benjamin Goldschmidt, Christoph Lerche, Peter Michael Dueppenbecker, Patrick Hallen, Bjoern Weissler, Pierre Gebhardt, Fabian Kiessling, and Volkmar Schulz

\begin{abstract}
In positron emission tomography (PET) systems, light sharing techniques are commonly used to readout scintillator arrays consisting of scintillation elements, which are smaller than the optical sensors. The scintillating element is then identified evaluating the signal heights in the readout channels using statistical algorithms, the center of gravity (COG) algorithm being the simplest and mostly used one. We propose a COG algorithm with a fixed number of input channels in order to guarantee a stable calculation of the position. The algorithm is implemented and tested with the raw detector data obtained with the Hyperion-II ${ }^{D}$ preclinical PET insert which uses Philips Digital Photon Counting's (PDPC) digitial SiPMs. The gamma detectors use LYSO scintillator arrays with $30 \times 30$ crystals of $1 \times 1 \times 12 \mathrm{~mm}^{3}$ in size coupled to $4 \times 4$ PDPC DPC 3200-22 sensors (DPC) via a 2-mm-thick light guide. These self-triggering sensors are made up of $2 \times 2$ pixels resulting in a total of 64 readout channels. We restrict the COG calculation to a main pixel, which captures most of the scintillation light from a crystal, and its (direct and diagonal) neighboring pixels and reject single events in which this data is not fully available. This results in stable COG positions for a crystal element and enables high spatial image resolution. Due to the sensor layout, for some crystals it is very likely that a single diagonal neighbor pixel is missing as a result of the low light level on the corresponding DPC. This leads to a loss of sensitivity, if these events are rejected. An enhancement of the COG algorithm is proposed which handles the potentially missing pixel separately both for the crystal identification and the energy calculation. Using this advancement, we show that the sensitivity of the Hyperion- $\mathrm{II}^{\mathrm{D}}$ insert using the described scintillator configuration can be improved by $20-100 \%$ for practical
\end{abstract}

Manuscript received September 29, 2014; revised January 20, 2015; accepted April 04, 2015. Date of publication May 01, 2015; date of current version June 12, 2015. This work was supported by the European Community Seventh Framework Programme, project number 241711, and SUB nanosecond Leverage In PET/MR ImAging (SUBLIMA). The project "ForSaTum" is co-funded by the European Union (European Regional Development Fund Investing in your future) and the German federal state North Rhine-Westphalia (NRW). The Centre of Excellence in Medical Engineering funded by the Wellcome Trust and EPSRC under Grant WT 088641/Z/09/Z

D. Schug, J. Wehner, B. Goldschmidt, and P. Hallen are with the Department of Physics of Molecular Imaging Systems, Institute for Experimental Molecular Imaging, RWTH Aachen University, Aachen, 52056, Germany.

P. M. Dueppenbecker and P. Gebhardt are with the Department of Physics of Molecular Imaging Systems, Institute for Experimental Molecular Imaging, RWTH Aachen University, Aachen, 52056, Germany and also with the Division of Imaging Sciences and Biomedical Engineering, King's College London, London, WC2R 2LS, U.K.

B. Weissler and V. Schulz are with the Department of Physics of Molecular Imaging Systems, Institute for Experimental Molecular Imaging, RWTH Aachen University, Aachen, 52056, Germany and also with the Department of Clinical Application Research, Philips Research, Aachen, 52056, Germany.

C. Lerche is with the Department of Oncology Solutions, Philips Research, Eindhoven, 5656, Netherlands.

F. Kiessling is with the Institute for Experimental Molecular Imaging, RWTH Aachen University, Aachen, 52056, Germany.

Color versions of one or more of the figures in this paper are available online at http://ieeexplore.ieee.org.

Digital Object Identifier 10.1109/TNS.2015.2420578 useful readout thresholds of a single DPC pixel ranging from 17-52 photons. Furthermore, we show that the energy resolution of the scanner is superior for all readout thresholds if singles with a single missing pixel are accepted and correctly handled compared to the COG method only accepting singles with all neighbors present by $0-1.6 \%$ (relative difference). The presented methods can not only be applied to gamma detectors employing DPC sensors, but can be generalized to other similarly structured and self-triggering detectors, using light sharing techniques, as well.

Index Terms-Data processing, gamma-ray detectors, positron emission tomography (PET), PET instrumentation, scintillation detectors, semiconductor devices, signal processing, time-of-flight PET.

\section{INTRODUCTION}

$\mathbf{P}$ OSITRON EMISSION TOMOGRAPHY (PET) is a functional imaging modality with high sensitivity. To detect the two annihilation photons (in the following referred to as gammas) from a positron annihilation, traditional PET detectors employ scintillators converting the single $511 \mathrm{keV}$ gamma to a few thousand optical photons which are detected using photosensors such as photomultipliers, avalanche photodiodes (APD) or silicon photomultipliers (SiPM). If the pitch of the scintillator elements (referred to as crystal) is smaller than the pitch of the readout channels, a one-to-one coupling of readout channel and crystal is not feasible. One common solution is to use a light sharing element to distribute the optical photons from a single crystal to more than one readout channel. The signal distribution can then be used to deduce the crystal in which the scintillation light has been produced.

Our group developed a PET insert based on digital silicon photomultipliers (dSiPM) which is designed to be operable in a 3-T magnetic resonance imaging (MRI) system. The underlying platform employing analogue SiPMs, Hyperion-I, has been evaluated in [1]. The successor of the platform, Hyperion-II ${ }^{\mathrm{D}}$, which is used in this work, has first been presented in [2]-[4].

Digital SiPMs digitize the breakdown of individual single photon avalanche diodes (SPAD) directly on sensor level, meaning that no further digitization steps are needed. This makes the dSiPM less prone to variations in the signal height due to temperature or voltage fluctuations. Philips Digital Photon Counting (PDPC) presented the first dSiPM in 2009 [5]-[7]. In this work, we are using a photodetector readout based on PDPC digital photomultiplier DPC 3200-22 (DPC) sensors which have $2 \times 2$ channels (also referred to as pixels) per sensor. 
DPCs are already widely used to investigate potential applications of dSiPMs for PET and SPECT [8]-[17]. All investigations are based on evaluation boards provided by PDPC, namely the Technology Evaluation Kit (TEK).

In this paper, we describe the techniques used to process raw DPC hit data to PET singles information on the Hyperion- II $^{\mathrm{D}}$ scanner. The techniques presented here can be translated to a wide range of other systems using segmented scintillators coupled to sensor arrays with digitization and self-triggering per channel employing a light sharing element. This is especially the case for systems based on the TEK using the same DPC technology as the presented system.

The center-of-gravity (COG) or Anger algorithm has been widely used as a crystal identification algorithm for detectors using a light sharing element. For analog detectors, it can be implemented using a resistive network for signal summation [18]. If the light pulse is distributed only over a small fraction of the total number of channels of a readout sensor array, the noise of sensors located far away from the position of the scintillation event deteriorates the calculated COG. Therefore, advanced COG algorithms try to give the channels closest to the event a higher weight compared to channels capturing none or only a small fraction of the scintillation pulse or they define a region-of-interest (ROI) around the readout channels with the highest signal. In a purely analogue implementation of a COG this can be realized by an offset subtraction in each channel. It has been shown that the crystal identification can be improved by such a subtraction [19], [20].

If the channel values are digitized and processed individually, more advanced algorithms can be implemented. One method to reduce the noise channel contribution is an intensity-weighted COG [21]. Another possibility is to use a ROI to restrict the channels used for the COG calculation [22]. These algorithms can benefit from an iterative formulation [23] at the expense of increased computation time.

Nevertheless, an unrestricted COG is commonly used on systems with digital information available per readout channel [15]. Using the PDPC TEK and its capability to trigger all readout channels, one can restrict to calculate the COG only if all channels are present, which leads to a stable number of input values. As it mimics the simple analogue $\mathrm{COG}$, it is still very prone to noise and reduces the sensitivity of the gamma detector due to requiring all channels to have been read out. A subtraction algorithm implemented for digitized data acquired with the TEK shows improvements of the COG stability [15]. Using no light guide but transparent coupling between crystals on a $2 \times 2$ channels DPC sensor, the COG calculation can be restricted to the four channels of this DPC [24].

Our group presented a stable ROI filter, which was used for the energy calculation, in 2011 [25]. In this paper, we will present a further improved version of a COG algorithm with a stable ROI selection. In order to improve the sensitivity, we show an enhancement of the algorithm to accept up to two ROIs or sets of channels for each crystal.

Besides a COG approach, there are more advanced algorithms which can be used for crystal identification, like for example a maximum-likelihood identifier [26]. These algorithms can handle changing sets of input channels for the same

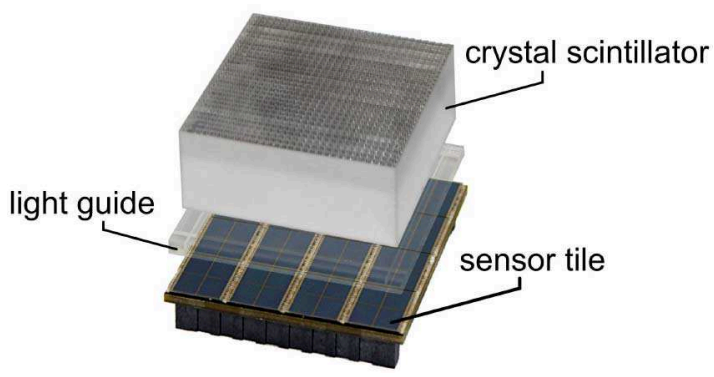

Fig. 1. Exploded photo of the arrangement of a detector stack. The reflective film on top of the crystal array has been removed to show the individual crystals. In the final scanner, after the components have been glued together, the detector stack is wrapped with Teflon tape to close up the optically transparent light guide.

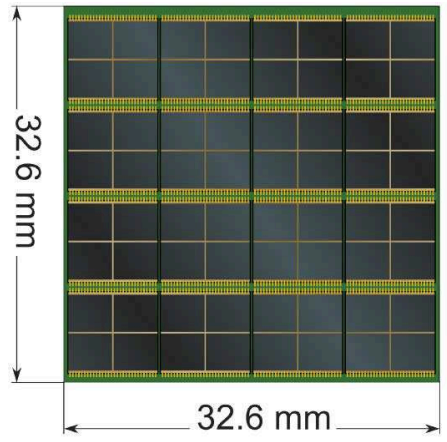

(a)

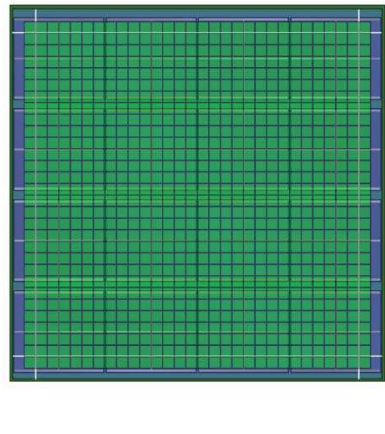

(b)
Fig. 2. (a) Sketch of the sensor tile. (b) Sketch of the LYSO array coupled via a slitted lighguide onto the sensor tile.

crystal very robustly but are computationally more complex and require a more elaborate calibration scheme than a COG approach. They are not in the scope of this paper.

\section{MATERIALS}

The Hyperion-II ${ }^{\mathrm{D}}$ platform is described in detail in [2]. In this work we will only describe the relevant components needed to explain the data processing.

\section{A. Detector Stack}

The detector stack consists of a scintillation crystal array, a light guide, a sensor tile which is used to read out the scintillation light and an FPGA-based control and readout interface board [27] (Fig. 1).

\section{B. Sensor Tile and PDPC DPC 3200-22 sensor}

The sensor tile is $32.6 \times 32.6 \mathrm{~mm}^{2}$ in size and is made up of 16 DPCs (PDPC DPC 3200-22) [5]-[7] (Fig. 2(a)).

DPC Layout: DPCs provide a digital photon count per readout channel and a single time stamp. A DPC comprises $2 \times 2$ pixels consisting of 3200 SPADs each. When an avalanche is induced in a SPAD, the voltage drop is detected and stored as binary information. An active quenching circuit stops the avalanche. When a readout is requested, all SPAD breakdowns are summed up. SPADs that show a significantly high dark count rate (DCR) can be inhibited individually.

Trigger and Validation Hardware Implementation: A pixel is further divided into four sub-pixels. The 800 SPADs per sub- 


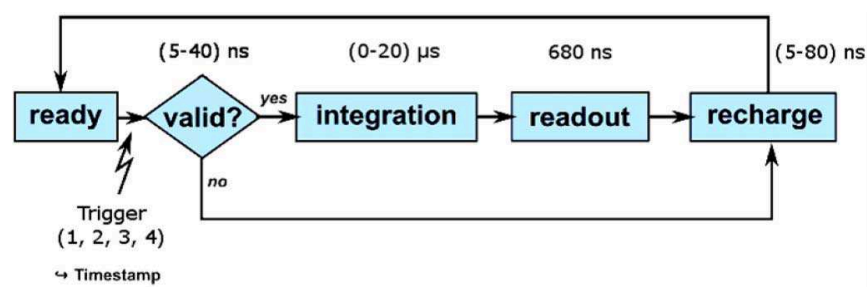

Fig. 3. Sketch of the DPC trigger, validation and readout state machine (from [28], courtesy of PDPC).

pixel are grouped together for the generation of a trigger signal. 32 SPADs form a row trigger line (RTL). There are 25 RTLs per sub-pixel. The trigger signals of the RTLs are used for a fast and low-threshold trigger generation and for the validation network which is used as a higher readout threshold to discard noise triggers. While for the trigger mechanism all RTLs of a sub-pixel are combined with OR gates, the validation network can be programmed for different logical combinations of the RTLs of a sub-pixel. Details can be found in [9] and [28].

The readout state machine of the DPC is shown in Fig. 3. We will explain the involved steps in the following.

Trigger: A trigger scheme can be set between 1-4. The trigger scheme 1 generates a trigger on the first measured breakdown of a SPAD in any of the four sub-pixels. The higher schemes 2, 3 and 4 are logical operations of the four sub-pixel trigger lines requiring $2.33 \pm 0.67,3.0 \pm 1.4$ and $8.33 \pm 3.80$ mean number of SPAD breakdowns assuming a homogeneous distribution of SPAD breakdowns on a pixel to reach the trigger condition, respectively [9]. The trigger lines of the four pixels of a DPC are connected by an OR gate.

If trigger schemes higher than 1 are used, RTL noise triggers will accumulate over time and generate a noise trigger. To overcome this limitation, the optional RTL refresh feature recharges single RTLs that generated a trigger after 10-15 ns of its occurrence if subsequently the global DPC trigger is not reached [5].

When the trigger condition is reached, a time stamp is generated.

Two time-to-digital converters (TDC) are running at half the DPC's clock speed, which is $200 \mathrm{MHz}$, with a phase shift of half a TDC clock cycle with respect to each other (Details of the TDC design are discussed in [6]). Each uses a tapped delay line (TDL) with 512 bins and a bin width of approximately $20-25$ ps. The TDLs are used to generate a high resolution time stamp.

Validation: A trigger causes the DPC to enter a validation phase of programmable length (VL). The logic condition set in the configurable validation network (val) has to be fulfilled during the validation phase in order to validate a trigger (Fig. 3). The validation network uses 7 bits to connect trigger groups of 3-4 RTLs of a sub-pixel using logical operations (Fig. 4). A further bit defines the logical operation of the four sub-pixels. In this paper, we use a hexadecimal notation of the configuration of the validation network. The validation lines of the pixels on a DPC are connected using an OR gate. Assuming a uniform photon distribution, thresholds for the required number of registered photons for different validation network settings used in this work are listed in Table I.

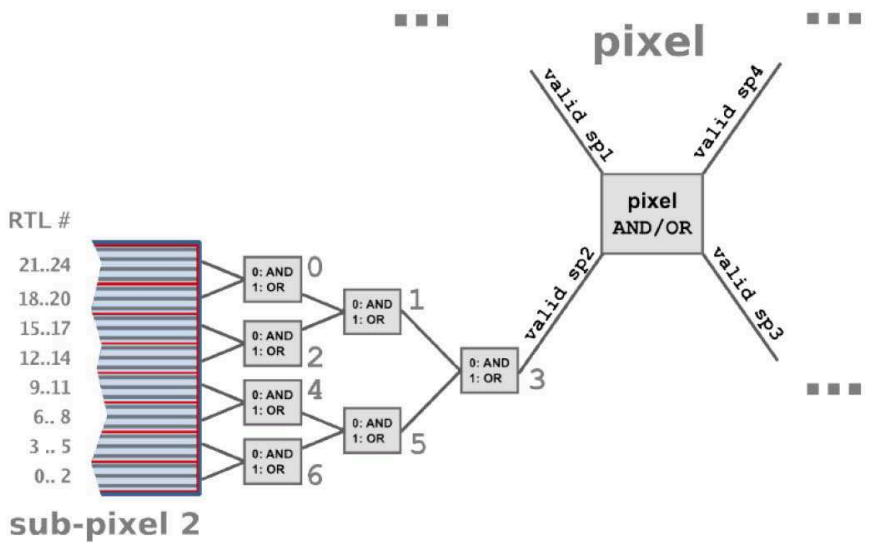

Fig. 4. Validation network of DPC 3200. The 7 bits of the validation network (val) can be set to configure a logical OR or AND for the combination of groups of 3-4 RTLs on a sub-pixel. A further bit sets the logical operation of the four sub-pixels. We state the configuration of the validation network in hexadecimal notation (Table I). (from [28], courtesy of PDPC).

TABLE I

DPC-3200-22 VALIDATION NETWORK IN HeXADECIMAL NOTATION AND THE Resulting Validation Threshold per PiXel (TAKen From [29], PARTLY PUblished IN [28] AND [9]). *ASYMMETRIC

\begin{tabular}{lrr}
\hline val & avg. number of SPADs & min number of SPADs \\
\hline $0 \times 55: \mathrm{OR}$ & $16.9 \pm 6.2$ & 4 \\
$0 \times 54: \mathrm{OR}^{*}$ & $27.5 \pm 10.3$ & 4 \\
$0 \times 50: \mathrm{R}^{*}$ & $37.1 \pm 12.8$ & 6 \\
$0 \times 00: \mathrm{OR}$ & $52.2 \pm 15.0$ & 8 \\
\hline
\end{tabular}

Integration and Readout: For a validated event an integration phase is started. This is used to accumulate SPAD breakdowns caused by the photon pulse. The integration length should be chosen long enough to capture the relevant part of the scintillation pulse. After integration, the readout is started and the binary information of each SPAD register is summed up giving the photon count per pixel. This readout is performed line by line and the lines which have not been read out yet are sensitive to optical photons also during this phase, so half of the readout time can be considered to contribute to the integration time.

After the consecutive recharge phase, all SPADs are sensitive again and the DPC is set back into the ready state waiting for the next trigger [5] (Fig. 3).

\section{Scintillator and Light Guide}

$30 \times 30$ cerium-doped lutetium yttrium orthosilicate (LYSO) crystals (Agile, Knoxville, USA) with a height of $12 \mathrm{~mm}$ and a pitch of $1 \mathrm{~mm}$ are mounted on a trenched $2 \mathrm{~mm}$ glass plate used for light spreading (Fig. 2(b)). The crystals are wrapped in $67 \mu \mathrm{m}$ Vikuity ESR film (3M, St. Paul, USA).

\section{Singles Detection Module}

Up to $2 \times 3$ detector stacks are mounted on a singles-detection module (SDM). A central synchronization unit, that distributes reference clocking and trigger signals to all SDMs, is used. The insert is composed of ten fully equipped SDMs mounted on a gantry. They form a PET scanner with a crystal-to-crystal spacing of $209.6 \mathrm{~mm}$ and an axial field of view of $97 \mathrm{~mm}$. The SDMs are cooled using a liquid cooling system and are flooded 
with dry air to allow a stable temperature control and prevent condensation.

\section{E. Data Acquisition and Processing}

An FPGA on the main SDM PCB handles the communication with the detector stacks [30]. Each SDM is connected via plastic optical fibers (POF) to a data acquisition and processing server (DAPS) [31]. An additional POF per SDM is connected to the synchronization unit. The DAPS either processes hit data in real time or stores it for offline analysis on hard disks. It is controlled via a control PC and routes the status and command communication between the PC and the SDMs.

\section{METHODS}

In this work, we use the full DPC raw data stored by the DAPS. An offline analysis is performed using a multi-threaded calibration and processing framework written in $\mathrm{C}++$.

Data processing consists of the following steps which are described in detail in this section. The data of each SDM is split into separate streams for each detector stack which can be processed in parallel. Raw hit data of a DPC is used to generate a time stamp and a corrected photon count. The hits are sorted, correlated and clustered according to their timestamps. Detector scatter between two stacks is not accounted for.

The resulting clustered data is used to identify the crystal that scintillated. The photon count of a subset of channels is used to calculate the energy deposited in the scintillator. Afterwards, coincidences are searched in the combined singles stream of all stacks.

\section{A. Raw DPC Hit Data Processing}

To calibrate the TDCs, we assume equally distributed triggers over a DPC clock cycle in order to calibrate the TDL bin widths. After the individual TDL calibration, assuming a fixed offset of half a clock cycle, the two TDCs are lineary calibrated against each other.

This calibration should be applied for each operating point since the TDLs change their bin width as a function of temperature.

A simple exponential saturation model is used to correct the photon count of each pixel taking the inhibited fraction of SPADs into account.

Differences of the effective photon detection efficiency (PDE) between pixels on the same sensor tile may occur. This is caused by slight variations in the break down voltage of DPCs or by coupling defects (e.g. glueing problems) between the light guide and the DPCs. Differences in the effective PDE can lead to an ambiguous relation of a crystal and the readout pixel capturing most of its scintillation light. As described later, this relation is essential for the COG ROI selection. If the effective PDE is not accounted for it can lead to distortions of the floodmap or even multiple COG positions for a single crystal due to an ambiguous ROI selection. The effective PDE of a pixel is estimated using the individual saturation-corrected photon data per pixel for non-clustered DPC hit data (method described in [32] and mentioned in [33]). The estimation has been performed with ${ }^{22} \mathrm{Na}$ data as well as FDG measurements and yields similar results.

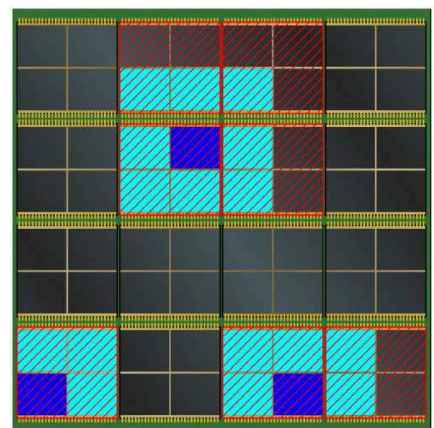

(a)

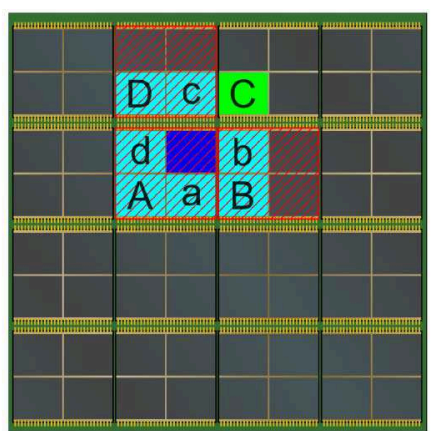

(b)
Fig. 5. (a) The three different scenarios of the main pixel position on a sensor tile are shown. A main pixel in the middle, at the edge and the corner. The main pixel is marked in dark blue, the surrounding neighbors used for the COG-FN calculation are shown in light blue. The DPCs used for E-FN energy calculation are marked in red. (b) A single missing the corner DPC. The COG-DN uses only the main pixel and the pixels depicted in light blue. The green pixel is linearly extrapolated using the other pixels to correct the calculated COG position for the COG-DN-Ex algorithm. For the given labeling of the pixels equation (2) gives the extrapolation formula used to calculate the missing pixel information depicted as $\mathrm{C}$. The energy using the E-DN method is calculated using the three DPCs present in the single. The energy calculation factor for this scenario may differ from the one using all four DPCs and the E-FN method. If the COG-DN and COG-DN-Ex are applied to singles with the corner DPC present, the information is discarded.

The spectrum $H(i)$ lists the frequency of the photon value $i$. For each photon value $i$ of the spectrum, we calculate the integral of photon value frequencies with at least $i$ photons. These integral values are logarithmized and then normalized to the logarithmized integral of the whole spectrum $(i=0)$. These monotonically descending functions $\mathrm{lsum}_{i}^{\prime}$ are used to define a stable high photon value per pixel via threshold $t$ as a measure for the effective PDE: $\operatorname{lsum}_{i(t)}^{\prime}=t$.

The threshold $t$ was empirically chosen to be 0.5 because values $\lesssim 0.3$ have proven to be prone to low statistic and therefore noisy high photon values. All effective PDE values of pixels are normalized to the system wide median. The linear effective PDE factor is applied after the saturation correction resulting in a corrected photon count.

\section{B. Clustering and Singles Processing}

It takes up to several nano seconds to fulfill the trigger condition on DPCs capturing only a small fraction of the scintillation light. Therefore, a fixed cluster window of $40 \mathrm{~ns}$ is chosen to find timely correlated hits on a sensor tile and combine them into singles. The timestamp of a single is defined as the timestamp of the earliest hit. This is in most cases the DPC located directly beneath a crystal and therefore captures the largest fraction of light.

Classification of Singles: The pixel with the highest corrected photon count in a single is defined as the main pixel. Pixels adjacent to the main pixel are called neighbor pixels. We distinguish between direct neighbors which are horizontally and vertically directly adjacent to the main pixel and the diagonal neighbors.

Singles are classified into two classes according to the presence of the neighboring pixels (Fig. 5).

The set of singles with all neighbor pixels present is depicted as FN and the set of singles with all direct neighbors present as DN. A further set, depicted as DNe, only includes the singles 
belonging to $\mathrm{DN}$ but not to $\mathrm{FN}$, these singles miss a diagonal neighbor pixel. Due to the $2 \times 2$ pixel layout of the DPCs there is a maximum of one DPC housing a diagonal neighbor pixel which is allowed to miss for a single to be an element of DN (Fig. 5(b)). The relations between the sets are given in equation (1).

$$
\mathrm{FN} \subseteq \mathrm{DN}, \quad \mathrm{FN} \cap \mathrm{DNe}=\emptyset, \quad \mathrm{DN}=\mathrm{FN} \cup \mathrm{DNe}
$$

To be a member of FN, singles need to contain the hit data of a varying number of DPCs depending on the position of the main pixel: one DPC for a corner pixel, two DPCs for an edge pixel and four DPCs for one of the central pixels.

If the main pixel of a single is located at the corner or at the edge of the sensor tile, all its neighbors are either located on the same DPC as the main pixel or on a second DPC that includes at least one direct neighbor. Therefore, these singles are always a member of FN and can not be a member of DNe (Fig. 5).

COG Calculation: The proposed COG algorithms operate on the main pixel and neighbor pixels with filtering for different classes of singles.

The COG algorithm which handles singles that are a member of FN, but not $\mathrm{DNe}$, and which uses the main and all neighbor pixels is called COG with full neighborhood (COG-FN) (Fig. 5(a)). This strict quality criterion leads to a loss of sensitivity, especially for high validation thresholds and for crystals located close to the center of a DPC as shown in the results.

A second algorithm recovers this sensitivity loss by accepting singles which are a member of $\mathrm{DN}$, which includes singles that are a member of DNe. For central main pixels this means a single diagonal neighbor pixel can be missing or is omitted. The algorithm is called COG-DN and the resulting positions for crystals (see result Fig. 6 COG-DN) are different from the positions obtained with the COG-FN algorithm (see result Fig. 6 COG-FN).

The difference of positions between the two algorithms can be handled either by using a lookup table (LUT) for each algorithm, which might be a disadvantage on platforms with restricted memory, or by correcting the positions of the COG-DN to match those of the COG-FN algorithm.

Possible implementations of a correction algorithm could be a purely geometrical calculation rule or an algorithm which uses the measured channel information to calculate the missing pixel information.

We decide to use a transformation method based on the measured photon information available and only use a single 2D LUT for both algorithms in this work. This reduces the calibration complexity and reduces the amount of calibration data. We transform the COG-DN positions by linearly extrapolating the missing pixel's photon count (COG-DN-Ex). If the situation in Fig. 5(b) is assumed the calculation, using the corrected photon values of each channel, is given in (2).

$$
C=0.5\left(0.5\left(\frac{c}{a}+\frac{D}{A}\right) B+0.5\left(\frac{b}{d}+\frac{B}{A}\right) D\right)
$$

This estimates the positions of crystals similar to the positions obtained with the COG-FN method (see result Fig. 6 COGDN-Ex) and allows us to use a single LUT for the COG-FN and COG-DN-Ex.

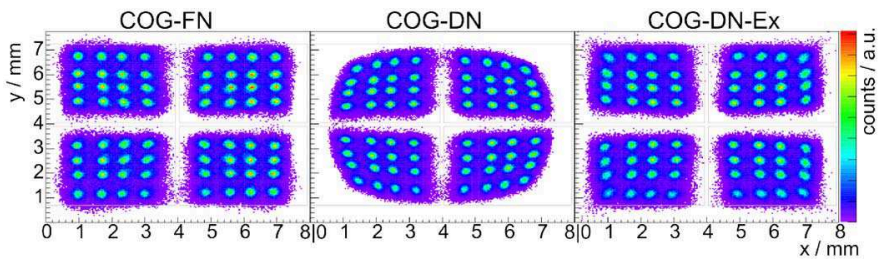

Fig. 6. COG positions for $8 \times 8$ crystals located over a central DPC calculated using the COG-FN (left), the COG-DN (middle) and the COG-DN-Ex (right) algorithm for a measurement using trigger scheme 4 and val $=0 \times 55$ : OR.

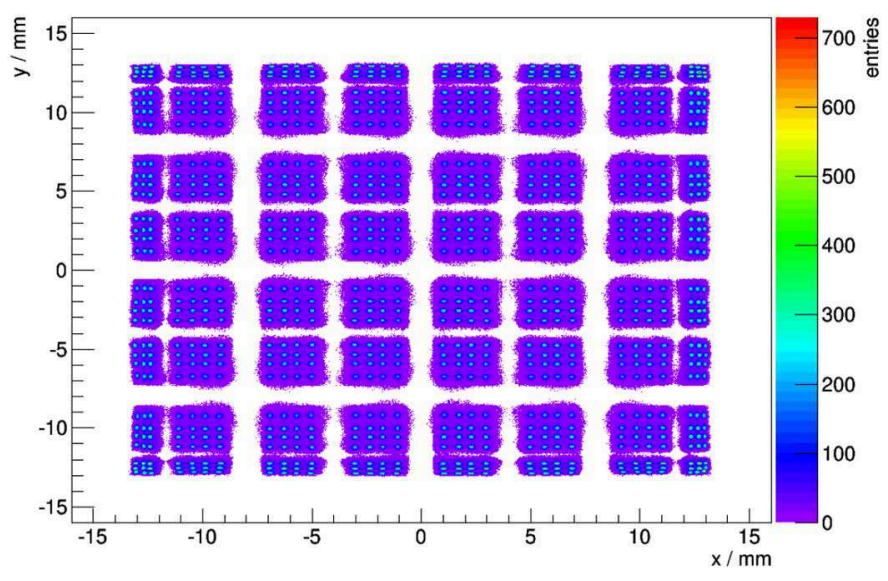

Fig. 7. Two-dimensional flood histogram of the COG-ACE for singles in the $\mathrm{NE}$ for a measurement using trigger scheme 3 and val $=0 \times 54: 0 \mathrm{R}$.

The sensitivity gain evaluated in this work is independent of how the DNe events are handled. A performance evaluation of the chosen correction algorithm and possible further distortion correction algorithms is beyond the scope of this work.

To get the best results, we applied the COG-FN for singles which are a member of FN and the COG-DN-Ex algorithm only for singles that are a member of DNe. We call this method COG with adaptive corner extrapolation (COG-ACE).

To extract the COG crystal position LUT, the COG-ACE positions for singles are filled into a 2D flood histogram per detector stack. Besides requiring that singles are a member of DN, we apply only a photon count filter of at least 400 corrected photons. The crystal positions of all $30 \times 30$ crystals are then being extracted from this flood histogram as described in the following.

As there is a fixed mapping between a crystal and its main pixel, the floodmap is divided into 64 areas containing only COG positions above a single pixel. Using a background removal and deconvolution algorithm [34]-[36] the 9-16 peaks are identified (see result Fig. 7). These positions are used to map the calculated COG of a single to a crystal using a nearest position search. This can either be done by calculating the distance of a single's COG to all crystal positions or by using a precalculated 2D LUT. Using a 2byte crystal ID the LUT on a $25 \mu \mathrm{m}$ pitched grid is approximately $2.4 \mathrm{MB}$ in size. The resolution of the LUT can be reduced further if the algorithm is implemented on a platform with restricted memory. The calculation of the COG and the lookup using a precalculated 2D LUT is computationally inexpensive compared to complex statistical classification algorithms. 
Energy Calibration and Calculation: For a single that is a member of FN, the energy can be calculated using all DPCs housing the main pixel and all neighbor pixels (E-FN) (Fig. 5(a)). If the single is a member of DN, a second energy calculation can be performed with only the requested DPCs (E-DN) (Fig. 5(b)). The latter method is the only one that can be applied for singles that are a member of DNe. To obtain the best possible energy resolution, the energy can be calculated using the E-FN method for singles which are a member of FN, and using the E-DN method if the single is a member of DNe. This adaptive energy calculation method is depicted as E-FD.

For each crystal a corrected photon spectrum is generated separately for E-FN and for E-DN. In the range of 500-3000 corrected photons the background is calculated and removed from the spectrum [37]-[39]. A peak search is performed using a Markov chain algorithm [35]. All found peaks are fitted with a Gaussian function. If more than one peak is found and successfully fitted, the highest peak is selected. For the energy calculation, the photo peak is set to an energy value of $511 \mathrm{keV}$. No baseline offset is considered. Thus, two different energy calibration factors are obtained, one for E-FN and one for E-DN.

Singles Filtering: The software framework allows singles filtering at all stages of the processing (e.g. prior or after coincidence processing). As we use the full raw hit data we can define a wide range of filters, e.g. energy, photon counts, presence of channels and more. We use a singles filter before the coincidence processing in order to reduce the amount of data which is forwarded to the coincidence search. Using the described COG algorithms, we ask for all channels needed for the COG calculation to be present discarding all other singles. We use two different energy windows: the narrow energy window (NE) selects singles around the photo peak and ranges from $411-561 \mathrm{keV}$, whereas the wide energy window (WE), commonly used in preclinical studies, ranges from $250-625 \mathrm{keV}$.

\section{Coincidence Processing}

Singles of all sensor tiles are timely ordered and synchronized into a single processing pipeline for the whole scanner. During calibration, a sliding coincidence window (cw) of $12.5 \mathrm{~ns}$ is used to account for time offsets of SDMs, detector stacks and individual DPCs. This setting covers $\pm 5 \mathrm{~ns}$, which accounts for one clock cycle delay per element at $200 \mathrm{MHz}$, and includes a safety margin. Only coincidences of exactly two singles are accepted. A minimum distance of 5 sensor tiles in tangential direction between the two singles is requested.

Due to cable lengths, the individual SDMs, Stacks and DPCs in the system can have a systematic time offset with respect to each other. This constant offset needs to be calibrated and accounted for in order to measure the time difference of two singles as accurate as possible. Time alignment is performed with either point sources distributed along the $z$-axis of the scanner or a line source. For the time alignment of the sensors, we take only the 4 crystals located closest to the center of a pixel into account. Crystals above bond gaps and between two pixels are expected to have a larger time delay and worse timing resolution and thus will be discarded for the calibration of the DPC offsets. The timing difference for each measured pair of DPCs in the system is corrected for the known source position and filled into a histogram. In order for the minimization problem to be over-determined and thus get absolute delay values for the whole system, it is beneficial to vary the $x$ and $y$ position (coordinates on the transversal plane) of point sources or place the line source slightly off center. This allows to measure coincidences to different stacks for a single stack. The source position is determined using a straight LOR and selecting the closest point source or the closest point on the line source. The mean value and its error is extracted from each histogram. A single delay value per DPC is fitted to all measured difference values using a least squares fit.

The same algorithm can be applied to fit delay values for individual crystals as an additional time offset correction. Fitting delay values of all 54000 crystals of the system poses challenges to the minimizer. A simplification is to only correct for a mean delay for each of the 900 crystal positions assuming that the mean delay value of a specific crystal position is a reasonable approximation for all detector stacks.

After time offset calibration the coincidence window is narrowed. This depends on the object size and the trigger scheme which influences the timing performance.

\section{Experiments}

We use five ${ }^{22} \mathrm{Na}$ point sources with $\sim 1.3 \mathrm{MBq}$ each and an FDG filled line source for the measurements shown in this work. We employ an overvoltage of $2.5 \mathrm{~V}$, inhibit $20 \%$ of the SPADS per DPC which show the highest DCR, use a validation length of $40 \mathrm{~ns}$, enable the RTL refresh feature and use an integration length of $165 \mathrm{~ns}$.

Energy resolutions for the system are given as FWHM of a Gaussian function fitted iteratively in the range of -0.5 to 1 FWHM around the photo peak of the coincident energy spectrum without background modeling.

\section{RESUlTs/DiscussiON}

Fig. 6 demonstrates that the COG-DN-Ex is able to correct the COG-DN positions reasonably well to match those of the COG-FN allowing to use a single LUT for both algorithms. The calculated $\mathrm{COG}$ positions for all crystals using the COG-ACE algorithm demonstrates that the COG-ACE algorithm is able to retain the separability of all crystal positions (Fig. 7).

The readout probability of the DPCs for a selected crystal located centrally on a sensor tile using the COG-ACE algorithm is shown in Fig. 8(a). As we only accept singles that are a member of DN, the readout probability for DPCs housing a direct neighbor pixel is $100 \%$. For the measurement taken with the WE, trigger scheme 3 and val $=0 \times 54$ :OR the readout probability of the DPC needed for the single to be a member of FN is $35 \%$. This high fraction of singles being a member of $\mathrm{DNe}$ is typical for crystals located close to the center of a single DPC. In Fig. 8(b) the mean corrected photon value for the same crystal is shown individually for each pixel. The mean corrected photon value is strongly biased by the energy window employed which is in this case the WE ranging from $250-625 \mathrm{keV}$. It can be seen, that for a centrally located crystal the neighbor DPCs carry only a small fraction of the total light of a single.

Plotting the ratio of singles being a member of FN to those being a member of $\mathrm{DN}$, one can observe the dependence of the 


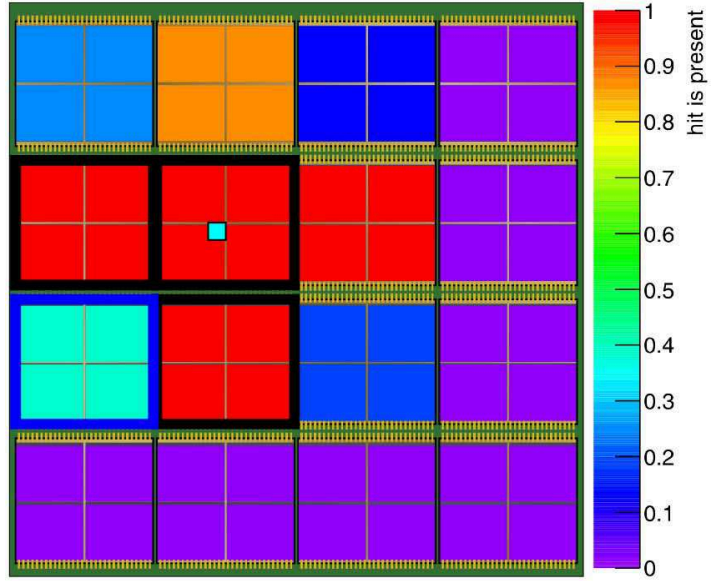

(a)

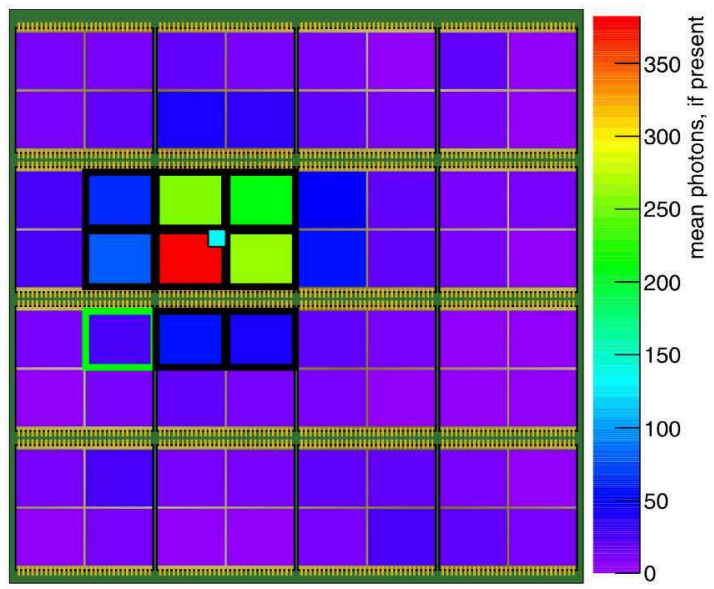

(b)

Fig. 8. The selected crystal, which is located centrally on the tile, is depicted in light blue. The measurement was taken using trigger scheme 3 and val $=0 \times 54: 0 R$. The data shown is plotted for coincident singles in a energy range of $250-625 \mathrm{keV}$ using the COG-ACE algorithm. (a) Readout probability as a function of the pixel position. The DPCs needed to be present in order for the single to be a member of DN are outlined in black, the additional DPC needed for the single to be a member of FN is outlined in blue. (b) Mean corrected photon value for readout events for each pixel. The mean corrected photon values of pixels far away from the crystal position, which have a low readout probability, are dominated by pile up and noise values. The pixels used for a stable COG-DN and COG-DN-Ex calculation are outlined in black. The additional pixel needed for the COG-FN calculation is outlined in green.

readout probability of the corner pixel on the crystal's position (Fig. 9(a)). For higher validation thresholds this effect intensifies (Fig. 9(b)). This ratio can be interpreted as a sensitivity loss when filtering for singles being a member of FN compared to a filter requiring singles to be a member of DN. Crystals located above the region where four DPCs adjoin show no sensitivity loss even for the highest validation threshold used. In contrast, crystals located close to the center of a single DPC show a sensitivity loss of about $30-40 \%$ for val $=0 \times 55: 0 \mathrm{R}$ which increases to almost $100 \%$ for val $=0 \times 00: 0 \mathrm{R}$ using the WE.

This translates into a relative loss of the system's sensitivity using the wide energy window for val $=0 \times 55: 0 \mathrm{R}$ and depending on the temperature of about $10-15 \%$. The relative sensitivity loss increases to $\sim 55 \%$ for val $=0 \times 00: 0 \mathrm{R}$ (Fig. 10). Higher temperatures introduce more thermal noise

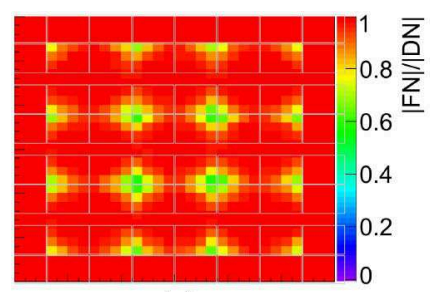

(a)

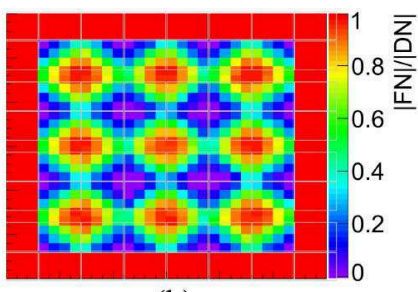

(b)
Fig. 9. Ratio of the cardinalities $|\mathrm{FN}| /|\mathrm{DN}|$ per crystal. The measurements were conducted at a cooling temperature of $5^{\circ} \mathrm{C}$ and trigger scheme 2 applying the wide energy window. Values are plotted for each crystal using the position in the crystal array. One can clearly see the location of the centers of DPCs, where the light has to propagate a longer path to reach the diagonal pixels. The fraction of events missing the corner DPC at high validation thresholds $(\mathrm{val}=0 \times 00: 0 \mathrm{R})$ (b) is higher compared to a low validation threshold $(\mathrm{val}=0 \times 55: 0 \mathrm{R})(\mathrm{a})$.

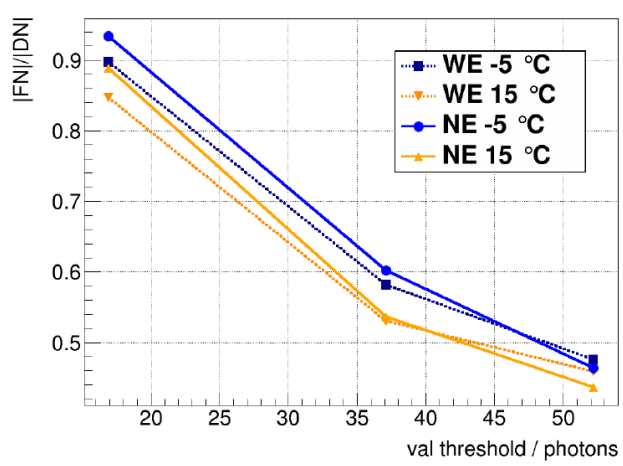

Fig. 10. Ratio of the crystal sensitivity of the single filter asking for all neighbors to be present to the filter allowing a missing corner pixel for the insert. The measurements were conducted at a cooling temperature of $-5^{\circ} \mathrm{C}$ and $15^{\circ} \mathrm{C}$ and trigger scheme 2 as a function of validation threshold. Lines are plotted to guide the eye.

to the system and thus lead to more dead time resulting in the corner DPC to be missing more often.

Depending on the geometrical position of the crystal - towards the edge of the sensor tile, above the center of a DPC or above a bond gap - the $511 \mathrm{keV}$ peak can be found at different corrected photon count values (Fig. 11(a)). Edge crystals and crystals located over bond gaps show a smaller corrected photon count of about 700-1200 corrected photons and a slightly worse energy resolution of $13-14.5 \%$ compared to more than 1600 corrected photons and an energy resolution of $11-14 \%$ for crystals centrally located over DPCs (Fig. 11(b)). Corner crystals show the lowest corrected photon count and the worst energy resolution of $\sim 15 \%$.

The calibrated energy spectrum for all crystals of a detector stack is shown in Fig. 12(a). The linearity can be checked by the second peak of the ${ }^{22} \mathrm{Na}$ with a gamma energy of $1275 \mathrm{keV}$ which is only $\sim 5 \%$ below the expected value. For PET the linearity is important if energy resolutions are determined by fitting the $511 \mathrm{keV}$ peak.

The coincident energy spectrum of the whole scanner from a measurement with an FDG filled line source with an activity of 8.3 MBq is shown in Fig. 12(b). The COG-ACE and E-FD algorithms are used with a selection of singles which are a member of DN with a minimum photon value of 100 corrected photons. 


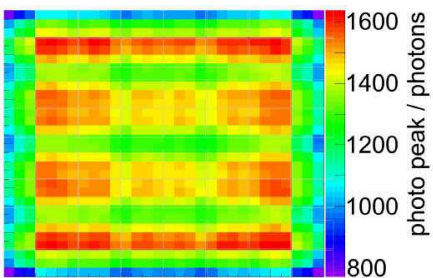

(a)

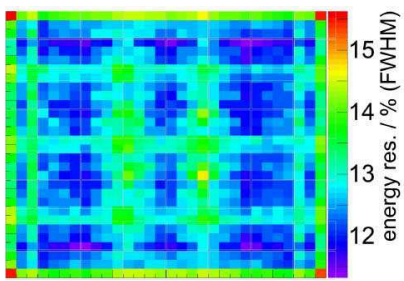

(b)
Fig. 11. Plots show the median value of the 60 detector stacks. Crystals located above the center of a DPC have a higher corrected photon value of the photo peak (a) and a better energy resolution (b) compared to those above bond gaps. Values are plotted for each crystal using the position in the crystal array. The calibration was performed with a measurement conducted at a cooling temperature of $5^{\circ} \mathrm{C}$, trigger scheme 2 and val $=0 \times 54: 0 R$.

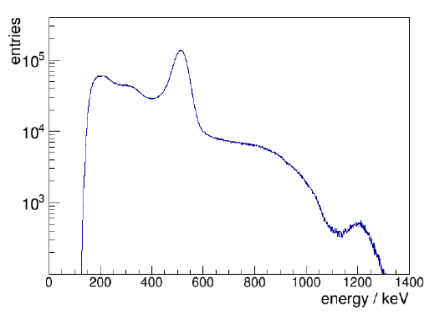

(a)

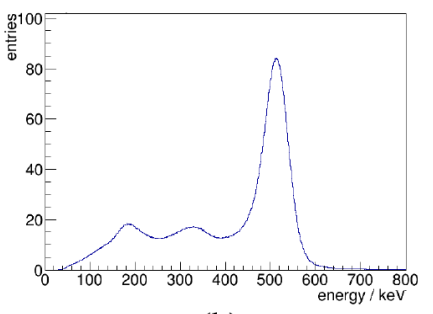

(b)
Fig. 12. (a) Singles energy spectrum for a calibrated detector stack. The ${ }^{22} \mathrm{Na}$ $1275 \mathrm{keV}$ peak can be seen at a slightly too low energy value. (b) Energy spectrum of coincident singles using only the neighbor filter and a minimum 100 corrected photons from a measurement with the FDG filled line source with an activity of $8.3 \mathrm{MBq}$. The photopeak at $511 \mathrm{keV}$ and the compton spectrum can be seen.

The scanner's energy resolution for coincident singles calculated using the different energy calculation algorithms is shown as a function of validation threshold in Fig. 13. All methods for energy calculation are close to each other and only differ by about $1.6 \%$ (relative difference). For E-DN the energy resolution stays stable for all validation thresholds at about $12.6 \%$. The energy resolution for E-FN starts at about $12.4 \%$ at low validation thresholds and degredates to the level of E-DN at high validation thresholds. This behavior can be explained by the bias of the filter requiring singles to be a member of FN. This filter has a higher efficiency for crystals located over bond gaps which have a worse energy resolution and thus increases their statistical weight (see Fig. 9(a), Fig. 9(b) and Fig. 11(b)). The E-FD method is able to maintain the energy resolution constantly at about $12.4 \%$. Thus, even allowing for data to be missing using the E-FD algorithm the overall system's energy resolution is not deteriorated. It even improves for higher validation thresholds and delivers the best system wide performance for the energy resolution of the three methods.

Timing offsets for the DPCs with respect to each other can be determined with an uncertainty of only a few pico seconds.

The delay between SDMs are caused by different signal run times in the synchronization lines and are in the order of a few hundreds of ps. The detector stacks on a SDM show a delay pattern with values in the order of $\pm 200 \mathrm{ps}$ with respect to each other. This pattern is similar for all SDMs as the firmware and signal paths are the same on all SDMs. For DPCs a similar pattern on all detector stacks can be observed with DPC delays in the order of $\pm 100 \mathrm{ps}$. The order of magnitude of these values

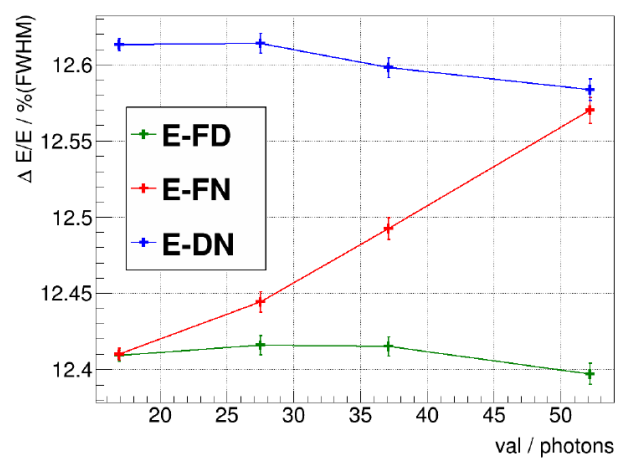

Fig. 13. Energy resolution as a function of the validation threshold for different energy calculation methods. The measurements were conducted at a cooling temperature of $15^{\circ} \mathrm{C}$ and trigger scheme 3 as a function of validation threshold. Lines are plotted to guide the eye.

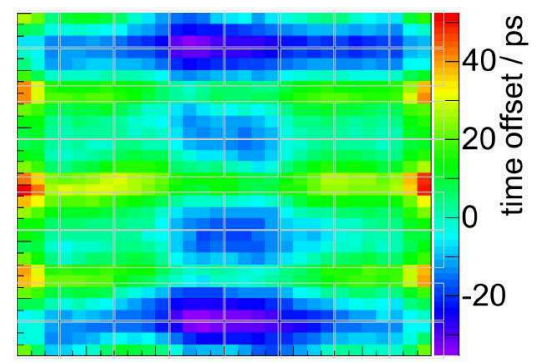

Fig. 14. Crystal time delays. Values are plotted for each crystal using the position in the crystal array.

shows the need to perform a time alignment down to the level of individual DPCs.

Even crystal delay values can be determined with a high accuracy but their absolute value is small compared to the timing performance of the scanner and is in the order of a few tens of ps and does not influence the overall timing performance (Fig. 14). The timing performance of the system, which is not discussed in detail in this paper, is roughly in the order of $260 \mathrm{ps}, 430 \mathrm{ps}$, $540 \mathrm{ps}$ and $1250 \mathrm{ps}$ for trigger settings $1-4$, respectively using 5 ${ }^{22} \mathrm{Na}$ sources, a cooling temperature of $-5^{\circ} \mathrm{C}$ and the NE. The timing performance of the system is not expected to change, if we allow a corner DPC to be missing, as it only captures a small fraction of the scintillation light and therefore has a long delay until the trigger is generated and does not influence the timestamp of a single.

\section{CONCLUSION/OUtLOOK}

For most crystals, the corner DPC captures the least scintillation light especially for crystals located close to the center of a DPC. Depending on the validation threshold, this leads to the corner DPC not being validated and missing in clustered singles data. Accepting singles with this DPC missing helps to improve the sensitivity of the scanner compared to only accepting singles with all neighbors present of up to a factor of two, depending on the validation threshold.

The presented extrapolation method COG-DN-Ex is able to correct the distorted COG position of the COG-DN method. Using a purely geometrical distortion corrections or a second 2D LUT are further possibilities to handle singles being a member of DNe for the crystal identification. If correctly accounted for, 
allowing the corner DPC to be missing improves the overall system energy resolution compared to filtering for singles with all neighbors present.

The presented time alignment procedure is able to accurately determine the systematic time offsets that can occur in the system and even allows to calibrate individual crystal offsets.

We present a robust DPC hit-to-single algorithm to obtain stable results regarding COG position and energy while maintaining a good sensitivity. This has been realized by accepting and correctly accounting for up to two sets of input channels per crystal. Especially high resolution applications, using a light sharing element, pose challenges due to low signal to noise levels of some channels. The proposed method is only slightly more complex than a simple COG method but still less computational expensive than more advanced crystal identification methods like a maximum likelihood algorithm. The model could be further enhanced to handle further sets of input channels.

The PET performance of the Hyperion- $\mathrm{II}^{\mathrm{D}}$ scanner is currently under investigation employing the described processing techniques.

\section{REFERENCES}

[1] B. Weissler, P. Gebhardt, C. W. Lerche, J. Wehner, T. Solf, B. Goldschmidt, J. E. Mackewn, P. K. Marsden, F. Kiessling, M. Perkuhn, D. Heberling, and V. Schulz, "MR compatibility aspects of a silicon photomultiplier-based PET/RF insert with integrated digitisation," Phys. Med. Bio., vol. 59, no. 17, p. 5119, 2014, 10.1088/0031-9155/59/17/ 5119.

[2] B. Weissler, P. Gebhardt, P. Dueppenbecker, B. Goldschmidt, A. Salomon, D. Schug, J. Wehner, C. Lerche, D. Wirtz, W. Renz, K. Schumacher, B. Zwaans, P. Marsden, F. Kiessling, and V. Schulz, "Design concept of world's first preclinical PET/MR insert with fully digital silicon photomultiplier technology," in Proc. IEEE NSS/MIC, Oct. 2012, p. 2113, 10.1109/NSSMIC.2012.6551484.

[3] J. Wehner, B. Weissler, P. Dueppenbecker, P. Gebhardt, D. Schug, W. Ruetten, F. Kiessling, and V. Schulz, "PET/MRI insert using digital SiPMs: Investigation of MR-compatibility," Nuclear Instrum. Meth. Phys. Res, Section A: Accelerators, Spectrometers, Detectors Assoc, Equip., vol. 734, pp. 116-121, 2014, 10.1016/j.nima.2013.08.077.

[4] J. Wehner, B. Weissler, P. M. Dueppenbecker, P. Gebhardt, B. Goldschmidt, D. Schug, F. Kiessling, and V. Schulz, "MR-compatibility assessment of the first preclinical PET-MRI insert equipped with digital silicon photomultipliers," Phys. Med. Bio., vol. 60, no. 6, p. 2231, 2015, 10.1088/0031-9155/60/6/2231.

[5] T. Frach, G. Prescher, C. Degenhardt, R. de Gruyter, A. Schmitz, and R. Ballizany, "The digital silicon photomultiplier - Principle of operation and intrinsic detector performance," in Proc. IEEE NSS/MIC, Nov. 2009, pp. 1959-1965, 10.1109/NSSMIC.2009.5402143.

[6] T. Frach, G. Prescher, C. Degenhardt, and B. Zwaans, "The digital silicon photomultiplier - System architecture and performance evaluation," in Proc. IEEE NSS/MIC, Nov. 2010, pp. 1722-1727, 10.1109/ NSSMIC.2010.5874069.

[7] C. Degenhardt, P. Rodrigues, A. Trindade, B. Zwaans, O. Mülhens, R. Dorscheid, A. Thon, A. Salomon, and T. Frach, "Performance evaluation of a prototype positron emission tomography scanner using digital photon counters (DPC)," in Proc. IEEE NSS/MIC, Oct. 2012, p. 2820, 10.1109/NSSMIC.2012.6551643.

[8] D. Schug, P. M. Dueppenbecker, P. Gebhardt, B. Weissler, B. Zwaans, F. Kiessling, and V. Schulz, "First evaluations of the neighbor logic of the digital SiPM tile," in Proc. NSS/MIC, Oct. 2012, p. 2817, 10.1109/ NSSMIC.2012.6551642.

[9] V. Tabacchini, V. Westerwoudt, G. Borghi, S. Seifert, and D. Schaart, "Probabilities of triggering and validation in a digital silicon photomultiplier," J. Instrum., vol. 9, no. 06, p. P06016, 2014, 10.1088/17480221/9/06/P06016.

[10] H. T. van Dam, S. Seifert, and D. R. Schaart, "The statistical distribution of the number of counted scintillation photons in digital silicon photomultipliers: Model and validation," Phys. Med. Bio., vol. 57, no. 15, p. 4885, 2012, 10.1088/0031-9155/57/15/4885.
[11] D. Schug, F. Kiessling, and V. Schulz, "Fast and unbiased 3D calibration method of arbitrary scintillator based PET detectors," in Proc. IEEE NSS/MIC, Oct. 2013, pp. 1-4, 10.1109/NSSMIC.2013.6829084.

[12] S. Seifert, G. Van der Lei, H. T. Van Dam, and D. R. Schaart, "First characterization of a digital SiPM based time-of-flight PET detector with $1 \mathrm{~mm}$ spatial resolution," Phys. Med. Bio., vol. 58, no. 9, p. 3061, 2013, 10.1088/0031-9155/58/9/3061.

[13] H. T. van Dam, G. Borghi, S. Seifert, and D. R. Schaart, "Sub- 200 ps CRT in monolithic scintillator PET detectors using digital SiPM arrays and maximum likelihood interaction time estimation," Phys. Med. Bio., vol. 58 , no. 10 , p. $3243,2013,10.1088 / 0031-9155 / 58 / 10 / 3243$.

[14] C. Bouckaert, S. Vandenberghe, and R. Van Holen, "Evaluation of a compact, high-resolution SPECT detector based on digital silicon photomultipliers," Phys. Med. Bio., vol. 59, no. 23, p. 7521, 2014, 10.1088/0031-9155/59/23/7521.

[15] M. Georgiou, G. Borghi, S. V. Spirou, G. Loudos, and D. R. Schaart, "First performance tests of a digital photon counter (DPC) array coupled to a $\mathrm{CsI}(\mathrm{Tl})$ crystal matrix for potential use in SPECT," Phys. Med. Bio., vol. 59, no. 10, p. 2415, 2014, 10.1088/0031-9155/59/10/2415.

[16] S. España, R. Marcinkowski, V. Keereman, S. Vandenberghe, and R. Van Holen, "DigiPET: Sub-millimeter spatial resolution small-animal PET imaging using thin monolithic scintillators," Phys. Med. Bio., vol. 59, no. 13, p. 3405, 2014, 10.1088/0031-9155/59/13/3405.

[17] C. Casella, M. Heller, C. Joram, and T. Schneider, "A high resolution TOF-PET concept with axial geometry and digital SiPM readout," $\mathrm{Nu}$ clear Instrum. Meth. Phys. Res. Section A: Accelerators, Spectrometers, Detectors Assoc. Equip., vol. 736, pp. 161-168, 2014, 10.1016/j. nima.2013.10.049.

[18] H. O. Anger, "Scintillation camera," Rev. Scientific Instrum., vol. 29, no. 1 , pp. $27-33,1958,10.1063 / 1.1715998$.

[19] R. Wojcik, S. Majewski, B. Kross, V. Popov, and A. Weisenberger, "Optimized readout of small gamma cameras for high resolution single gamma and positron emission imaging," in Proc. IEEE NSS, 2001, vol. 3, p. 18211825, 10.1109/NSSMIC.2001.1008696.

[20] Y. Qi, M. J. Zhang, C. Zhao, and R. Wojcik, "Performance comparison of subtractive resistive readout with conventional resistive readout for a high-resolution compact gamma camera," in Proc. IEEE NSS, Oct. 2007, vol. 5, pp. 3725-3728, 10.1109/NSSMIC.2007.4436931.

[21] R. Pani, F. Vittorini, M. Cinti, P. Bennati, R. Pellegrini, S. Ridolfi, R. Scafè, S. L. Meo, M. Mattioli, F. Navarria, G. Moschini, A. Fabbri, E. D'Abramo, V. O. Cencelli, and D. Sacco, "Revisited position arithmetics for LaBr3:Ce continuous crystals," Nucl. Phys. B - Proc. Suppl. , vol. 197, no. 1, pp. 383-386, 2009, 10.1016/j.nuclphysbps.2009.10. 109, 11th Topical Seminar on Innovative Particle and Radiation Detectors (IPRD08), issn: 0920-5632.

[22] E. Netter, L. Pinot, L. Menard, M. A. Duval, B. Janvier, F. Lefebvre, R. Siebert, and Y. Charon, "The tumor resection camera (TReCam), a multipixel imaging probe for radio-guided surgery," in Proc. IEEE NSS/MIC, Oct. 2009, pp. 2573-2576, 10.1109/NSSMIC.2009.5402022.

[23] C.-Y. Liu and A. L. Goertzen, "Improved event positioning in a gamma ray detector using an iterative position-weighted centre-of- gravity algorithm," Phys. Med. Bio., vol. 58, no. 14, p. N189, 2013, 10.1088/ 0031-9155/58/14/N189.

[24] R. Marcinkowski, S. España, R. Van Holen, and S. Vandenberghe, "Optimized light sharing for high-resolution TOF PET detector based on digital silicon photomultipliers," Phys. Med. Bio., vol. 59, no. 23, pp. 7125-7139, 2014, 10.1088/0031-9155/59/23/7125.

[25] P. Dueppenbecker, S. Lodomez, R. Haagen, P. Marsden, and V. Schulz, "Investigation of a sub-millimeter resolution PET detector with depth of interaction encoding using digital SiPM single sided readout," in Proc. IEEE NSS/MIC, Oct. 2011, pp. 2252-2253, 10. 1109/NSSMIC. 2011.6152490.

[26] C. Lerche, T. Solf, P. Dueppenbecker, B. Goldschmidt, P. Marsden, and V. Schulz, "Maximum likelihood based positioning and energy correction for pixelated solid state PET detectors," in Proc. IEEE NSS/ MIC, Oct. 2011, pp. 3610-3613, 10.1109/NSSMIC.2011.6153679.

[27] P. Dueppenbecker, B. Weissler, P. Gebhardt, D. Schug, J. Wehner, P. Marsden, and V. Schulz, "Development of an MRI compatible digital SiPM based PET detector stack for simultaneous preclinical PET/ MRI," in Proc. IEEE NSS/MIC, Oct. 2012, pp. 3481-3483, 10.1109/ NSSMIC.2012.6551794.

[28] Philips Digital Photon Counting, PDPC-TEK User Manual v0.21 2014.

[29] A. Thon, personal communication, Philips Digital Photo Counting, Apr. 2012. 
[30] P. Gebhardt, B. Weissler, M. Zinke, F. Kiessling, P. Marsden, and V. Schulz, "FPGA-based singles and coincidences processing pipeline for integrated digital PET/MR detectors," in Proc. IEEE NSS/MIC, Oct. 2012, pp. 2479-2482, 10.1109/NSSMIC.2012.6551565.

[31] B. Goldschmidt, C. W. Lerche, T. Solf, A. Salomon, F. Kiessling, and V. Schulz, "Towards software-based real-time singles and coincidence processing of digital PET detector raw data," IEEE Trans. Nucl. Sci., vol. 60, no. 3, pp. 1550-1559, 2013, 10.1109/TNS.2013.2252193.

[32] C. W. Lerche, S. Lodomez, V. Schulz, and B. Weissler, "Apparatus and Method for the Evaluation of Gamma Radiation Events," Patent W0/2014/180734, Jan. 22, 2015.

[33] C. W. Lerche, J. Mackewn, B. Goldschmidt, A. Salomon, P. Gebbhardt, B. Weissler, R. Ayres, P. Marsden, and V. Schulz, "Calibration and stability of a SiPM-based simultaneous PET/MR insert," Nucl. Instrum. Meth. Phys. Res. Section A: Accelerators, Spectrometers, Detectors Assoc. Equip., vol. 702, pp. 50-53, 2013, 10.1016/j.nima.2012. 08.041 .

[34] M. Mariscotti, "A method for automatic identification of peaks in the presence of background and its application to spectrum analysis," Nucl. Instrum. Meth., vol. 50, no. 2, pp. 309-320, 1967, 10.1016/0029-554X(67)90058-4, ISSN: 0029-554X.
[35] M. Morháč, J. Kliman, V. Matoušek, M. Veselský, and I. Turzo, "Identification of peaks in multidimensional coincidence $\gamma$-ray spectra," Nucl. Instrum. Meth. Phys. Res. A, vol. 443, pp. 108-125, Mar. 2000, 10.1016/S0168-9002(99)01005-0.

[36] Z. Silagadze, "A new algorithm for automatic photopeak searches," Nucl. Instrum. Meth. Phys. Res. Section A: Accelerators, Spectrometers, Detectors Assoc. Equip., vol. 376, no. 3, pp. 451-454, 1996, 10.1016/ 0168-9002(96)00230-6, ISSN: 0168-9002.

[37] C. Ryan, E. Clayton, W. Griffin, S. Sie, and D. Cousens, "SNIP, a statistics-sensitive background treatment for the quantitative analysis of PiXE spectra in geoscience applications," Nucl. Instrum. Meth. B, vol. 34 , no. 3, pp. 396-402, 1988, 10.1016/0168-583X(88) 90063-8, ISSN: 0168-583X.

[38] M. Morháč, J. Kliman, V. Matoušek, M. Veselský, and I. Turzo, "Background elimination methods for multidimensional coincidence $\gamma$-ray spectra," Nucl. Instrum. Meth. Phys. Res. Section A: Accelerators, Spectrometers, Detectors Assoc. Equip., vol. 401, no. 1, pp. 113-132, 1997, 10.1016/S0168-9002(97)01023-1, ISSN: 0168-9002.

[39] D. Burgess and R. Tervo, "Background estimation for gamma-ray spectrometry," Nucl. Instrum. Meth. Phys. Res., vol. 214, pp. 431-434, Sep. 1983, 10.1016/0167-5087(83) 90612-9. 